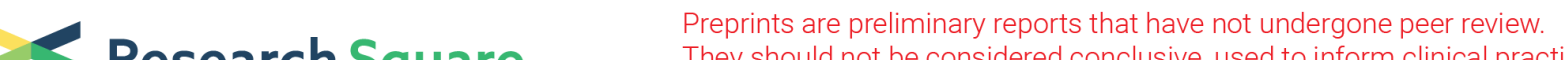 $\begin{array}{ll}\text { Research Square } & \begin{array}{l}\text { They should not be considered conclusive, used to inform clinical practice, } \\ \text { or referenced by the media as validated information. }\end{array}\end{array}$
}

\section{Novel mechanism of action for neuronal let-7b-5p in neonatal encephalopathy through the Hippo pathway}

Vennila Ponnusamy ( $\nabla$ vennilaponnusamy@nhs.net)

Blizard Institute, Barts and The London School of Medicine and Dentistry https://orcid.org/0000-00021987-5531

\section{Richard Ip}

Blizard Institute, Barts and The London School of Medicine and Dentistry

\section{Moumin Mohamed}

Blizard Institute, Barts and The London School of Medicine and Dentistry

\section{Paul Clarke}

Norfolk and Norwich University Hospitals NHS Foundation Trust

\section{Eva Wozniak}

Queen Mary University of London

\section{Charles Mein}

Queen Mary University of London

\section{Leslie Schwendimann}

French Institute of Health and Medical Research

\section{Akif Barlas}

The Royal London Hospital

\section{Philippa Chisholm}

Homerton University Hospital NHS Foundation Trust

\section{Ela Chakkarapani}

Bristol Medical School

\section{Adina Michael-Titus}

Queen Mary University of London

\section{Pierre Gressens}

Université de Paris https://orcid.org/0000-0002-0909-4221

\section{Ping Yip}

Queen Mary University of London

\section{Divyen Shah}

Blizard Institute, Barts and The London School of Medicine and Dentistry

\section{Article}


Keywords: microRNAs, neonatal encephalopathy, Hippo pathway

Posted Date: April 22nd, 2021

DOI: https://doi.org/10.21203/rs.3.rs-337473/v1

License: (c) (1) This work is licensed under a Creative Commons Attribution 4.0 International License. Read Full License

Version of Record: A version of this preprint was published at Communications Biology on September 30th, 2021. See the published version at https://doi.org/10.1038/s42003-021-02672-3. 


\section{Abstract}

Despite increasing knowledge on microRNAs, their role in the pathogenesis of neonatal encephalopathy remains to be elucidated. Herein, we identify let-7b-5p as a significant microRNA in neonates with moderate to severe encephalopathy from the dried blood spots using unbiased microRNA next generation sequencing. Validation studies using Reverse Transcription and quantitative Polymerase Chain Reaction on 45 neonates showed that let-7b-5p expression was increased on day 1 in neonates with moderate to severe encephalopathy with unfavourable outcome when compared to those with mild encephalopathy. Mechanistic studies performed on glucose deprived cell cultures and the cerebral cortex of two animal models of perinatal brain injury, namely hypoxic-ischaemic and intrauterine inflammation models confirm that let-7b-5p is associated with the apoptotic Hippo pathway. Significant reduction in neuronal let-7b-5p expression corresponded with activated Hippo pathway, with increased neuronal/nuclear ratio of Yes Associated Protein (YAP) and increased neuronal cleaved caspase-3 expression in both animal models. Similar results were noted for let-7b-5p and YAP expression in glucose-deprived cell cultures. Reduced nuclear YAP with decreased intracellular let-7b-5p correlated with neuronal apoptosis in conditions of metabolic stress. This novel finding of the Hippo-YAP-let-7b axis needs validation in larger cohorts and other models to further our knowledge on let-7b-5p as a biomarker for neonatal encephalopathy.

\section{Introduction}

Neonatal encephalopathy (NE) such as that associated with perinatal hypoxia-ischaemia remains an important cause of morbidity and mortality in term-born neonates globally ${ }^{1}$. Mild therapeutic hypothermia $(\mathrm{TH})$ has been shown to reduce death or severe neurodisability with a number needed to treat of seven to nine $e^{2,3}$. Improved understanding of the pathophysiology of NE is important in identifying reliable biomarkers and more effective treatments. Potentially microRNAs may have an important role in the pathophysiology of $\mathrm{NE}^{4}$.

MicroRNAs (miRNAs), a group of small non-coding RNAs made up of 17-22 nucleotides, are important regulators of a number of processes in health and disease, in conditions including cancer ${ }^{5}$, cardiovascular disease ${ }^{6}$, inflammatory disorders ${ }^{7}$ and immature brain injury ${ }^{4}$. There are relatively few studies on the role of miRNAs in NE, using neonatal blood samples ${ }^{8,9}$ and umbilical cord samples ${ }^{10,11}$. Hence in the present study, we set out to identify candidate miRNAs from blood samples of newborns with NE and to investigate the corresponding cellular and molecular mechanisms linked to these miRNAs through use of in vitro and in vivo models of NE.

In our analysis of miRNA next generation sequencing (NGS) of neonates with NE, the apoptotic Hippo signaling pathway was identified to be associated with a number of miRNAs that were differentially expressed between the favourable and unfavourable outcome groups, in particular the miRNA let-7b-5p. The Hippo signaling pathway is an important, well conserved pathway known to function across a number of mammalian species ${ }^{12,13}$ with a role in regulating apoptosis and cell proliferation. Within this pathway there are kinase complexes that negatively regulate the activities of the mammalian Yorkie (YKi) 
homolog named Yes Associated Protein (YAP) and transcriptional coactivator with PDZ-binding motif (TAZ).

The function of the Hippo pathway is dependent on the translocation of YAP/TAZ from the cytoplasm into the nucleus. In physiological conditions, the Hippo pathway is not activated so the YAP/TAZ complex remains unphosphorylated and is translocated into the nucleus. In the nucleus, YAP/TAZ bind to Transcription enhancer factor-1 (TEA/TEF) domain transcription factors 1-4 (TEAD 1-4) which are key DNA-binding platforms for YAP/TAZ to regulate cell growth in humans ${ }^{14}$. The nuclear YAP/TAZ/TEAD protein complex transcribes anti-apoptotic genes and maintains cell survival ${ }^{15}$. However, in pathological conditions such as stress, activation of the Hippo pathway leads to activation of YAP through phosphorylation. The phosphorylated YAP/TAZ remains in the cytoplasm and undergoes proteolytic degradation. In our study, we show using both in vitro and in vivo models of NE that neuronal apoptosis is associated with a decrease in let-7b-5p and a reduced nuclear location of YAP. This indicates a potential role of the Hippo pathway in the cell death associated with NE.

\section{Results}

\section{Identification of candidate and endogenous control miRNAs in moderate to severe neonatal encephalopathy using miRNA next generation sequencing}

From the four cohorts of neonates (1A: moderate to severe NE with $\mathrm{TH}$ and favourable outcome, 1B: moderate to severe NE with TH and unfavourable outcome, 2: mild NE without TH, 3: healthy cord blood, $\mathrm{n}=4$ per group) a total of 2795 miRNAs were identified in the miRNA NGS analysis performed using dried blood spots (DBS). Unsupervised data analysis using principal component analysis (PCA) did not reveal any unique clustering based on any of the simple parameters (i.e. time, injury or outcome) (Fig. 1a). A number of miRNAs were differentially expressed between the groups with unadjusted $p$ value $<0.05$ (Fig. 1b) and the heat map generated after correcting for false discovery rate (FDR) $<0.05$ revealed 17 significant miRNAs (Fig. 1C) which were differentially expressed in healthy cord blood compared to that of neonatal blood samples. On removing umbilical cord blood samples from the analysis, and correcting for FDR, there were no miRNAs that had a significant differential expression between the three remaining groups (Fig. 1d) as demonstrated in the heat map (Fig. 1e).

Among the neonates with NE, there was a pool of 134 significant miRNAs differentially expressed between mild NE and moderate to severe NE with favourable and unfavourable outcome groups. Of these, (top ten in descending order of significance), miR-29b-3p, miR-3200-3p, let-7b-3p, miR-3682-3p, miR-337-5p, miR-3200-5p, let-7b-5p, miR-412-5p, miR-4467 and miR-548 were noted to have the most significant $p$ value attributes. Using hierarchical clustering analysis, let-7b-5p, let-7b-3p and miR-3200-3p were identified as the most differentially expressed miRNAs between favourable and unfavourable outcome groups (Fig. 2). KEGG pathway analysis of all 134 miRNAs identified multiple pathways involving a number of miRNAs with $p$ values $<0.05$ (Fisher's exact test) after applying FDR correction. Since NE is associated with cell death, the apoptotic pathways were studied ${ }^{4}$. KEGG analysis identified 
three apoptotic pathways, namely the Hippo signaling pathway, P53 signaling pathway and Lysine degradation pathway (Supplemental Fig. 4). However, the only apoptotic pathway to differentially express both let-7b-3p and let-7b-5p between Group 1 neonates with favourable and unfavourable outcome was the Hippo signaling pathway. The use of reverse pathway analysis of the Hippo pathway confirmed let$7 b-5 p$ to be in the top ten most significant miRNAs of all the 771 miRNAs involved in this pathway. Using Normfinder, miR-454-5p was identified as a suitable endogenous control from 2795 miRNAs detected in the NGS analysis, with a good stability value of 0.68 . Following NGS data analysis, let-7b-3p, let-7b-5p and miR-3200-3p were studied for validation as candidate miRNAs along with miR-454-5p as endogenous control in the next step using Reverse Transcription and quantitative Polymerase Chain Reaction (RT-qPCR).

\section{Validation of control and candidate miRNAs through RT-qPCR}

Independent validation of the endogenous control and four candidate miRNAs was performed on the three neonatal subgroups ( $1 \mathrm{~A}, 1 \mathrm{~B}$ and $2 ; \mathrm{n}=15$ each) after excluding umbilical cord blood samples. Neonates in Group 1 (moderate to severe NE with TH) had a significantly lower Apgar score at 10 min, were more likely to require respiratory support and need chest compressions compared to those in Group 2 (mild NE without TH) as shown in Table 1. We have shown that there was a strong correlation between

MRI outcome and two-year neurodevelopmental outcome in this cohort ${ }^{16}$. Of the neonates with moderate to severe NE treated with $\mathrm{TH}$, all those in the favourable MRI group had a normal neurodevelopmental outcome as opposed to only one in the unfavourable MRI group at a median age of 2.5 years. Eleven children in the unfavourable MRI group developed cerebral palsy.

MiR-454-5p was validated as a reliable endogenous control miRNA with stable expression across all groups (Fig. 3a) and time points (S1 - Day 1, S2 - Day 2-3 and S3 - Day 5) (Fig. 3b). Let-7b-5p expression in $\mathrm{S} 1$ samples exhibited a significant increase in moderate to severe NE with $\mathrm{TH}$ and unfavourable outcome compared to mild NE without TH ( $p=0.019)$ (Fig. 3c), while there was no significant change in temporal expression in Group 1 neonates between the outcome groups or other time points (Fig. 3d). However, let-7b-3p and miR-3200-3p did not demonstrate any differential expression both at $\mathrm{S} 1$ between all 3 groups or on temporal expression data at different time points on S1, S2 and S3 samples (Fig. 3e to Fig. 3h). Because of the significant differential expression of let-7b-5p in neonates with NE, it was further studied in vitro and in two animal models of NE to further understand the mechanistic pathway.

\section{Reduced expression of let-7b-5p and YAP in glucose-deprived neuronal cells in vitro}

Let-7b-5p expression in neurones was studied in an in vitro NE model using cell cultures under glucose deprivation, to mimic metabolic stress. In comparison with control cell cultures, glucose-deprived cultures showed significantly reduced expression (mean+/- SEM) of total let-7b-5p immunostaining area (13.1 +/1.6 vs $5.6 \pm 0.6$ A.U., $p=0.004$ ) (Fig. $4 a$, Fig. $4 b$ ). More specifically, let-7b-5p expression in NeuN positive neuronal cells was significantly reduced $(50.78+/-1.36$ vs $11.23+/-1.10 \%$ area, $p<0.001)$ (Fig. 4 c) as 
was the percentage of neuronal let-7b-5p positive cells (99.96 +/- 0.04 vs $79.94+/-8.31, p=0.03)$, confirming that the majority of let-7b-5p positive cells was neuronal (Fig. 4d).

YAP, a core component of the Hippo signaling pathway, was studied in neuronal cells in the in vitro NE model as the KEGG analysis highlighted the significance of the apoptotic Hippo pathway in neonates with NE. Because a reduction in nuclear to cytoplasmic YAP expression is important to demonstrate an activated Hippo pathway, ${ }^{17}$ co-immunostaining of YAP and NeuN, with Hoechst staining of the nucleus, was carried out to identify the neuronal (cytoplasmic) and nuclear YAP expression separately, as clearly observed (Fig. 4e). Neuronal /nuclear YAP ratio (mean +/-SEM) in glucose-deprived neuronal cells was significantly higher $(2.32+/-0.07$ vs $1.67+/-0.08, p<0.001)$ when compared to control neuronal cells (Fig. 4f). This significant change in ratio was due to both a significant reduction (mean $+/$ - SEM) in nuclear YAP expression (21.21 +/- 0.81 vs $28.39+/-0.73, p<0.001)$ (Fig. 4g) and a significant increase in neuronal cytoplasmic YAP expression ( $50.1+/-1.84$ vs $44.16+/-1.07, p=0.007)$ (Fig. 4h) in glucosedeprived neuronal cells compared to neuronal cells in normal control conditions. These findings suggest activation of the Hippo pathway leading to increased neuronal YAP and reduced nuclear YAP, leading to reduced let-7b-5p expression under metabolic stress.

\section{Neuronal cell death in the cerebral cortex of rat neonatal encephalopathy models}

Two animal models of NE were studied as these may correspond to clinical NE in the human setting more closely. In the cerebral cortex, cleaved caspase-3 expression representing apoptotic cell death was significantly increased (mean +/- SEM) in hypoxic-ischaemic pups on the ipsilateral side $(45.9+/-0.4, p=$ $0.004)$, contralateral side $(51.9+/-3.6, p<0.001)$ and the intrauterine inflammatory model pups $(42.4+/-$ $1.1, p=0.006)$ when compared to control pups (30.0+/-3.4) (Fig. 5a, Fig. 5b). However, the total number of neuronal cleaved caspase- 3 positive cells was not significantly different between the animal models ( $p$ value $=0.37$, Fig. $5 \mathrm{c}$ ).

\section{Let-7b-5p expression in the rat neonatal encephalopathy models}

After confirming the presence of apoptosis in the NE models, let-7b-5p expression was studied in the cerebral cortex of both NE models in comparison to the control pups. Combined fluorescent in situ hybridisation (FISH) and immunohistochemistry (IHC) analysis using NeuN showed that let-7b-5p was predominantly expressed in cerebral cortical neurones (Fig. 6a). In the cerebral cortex, the percentage of neuronal let-7b-5p expression (mean $+/$ - SEM) in comparison to the control pups was significantly reduced in both ipsilateral $(69.9+/-2.3, p<0.001)$ and contralateral $(74.0+/-2.8, p<0.001)$ sides in the hypoxic-ischaemic model and the intrauterine inflammation model $(71.4+/-5.8, p<0.001)$ (Fig. 6b).

As for the human neonatal samples, let-7b-5p expression was studied in the DBS of peripheral blood of $\mathrm{NE}$ animal models to understand the peripheral expression pattern. The relative quantification of let-7b-5p normalised to miR-454-5p in the peripheral blood of rat pups showed that there was a significant decrease in expression (mean $+/$-SEM) in the intrauterine inflammation model $(0.28+/-0.09, p=0.022)$, but not hypoxic-ischaemic model $(0.90+/-0.27, p=0.86)$ when compared to the control $(1.0+/-0.17)$ (Fig. 6c). Using let-7b-3p as a negative miRNA control, the relative expression (mean $+/$ - SEM) for let-7b- 
$3 p$ expression in hypoxic-ischaemic model $(1.89+/-0.43)$ and intrauterine inflammation model $(1.89+/-$ 1.24) were not significantly different to the control $(1.31+/-0.46)$ (overall $p$ value $=0.90)$ (Fig. $6 \mathrm{~d}$ ). This suggests that there was an alteration in peripheral let-7b-5p expression after intrauterine inflammation. Similar to the in vitro NE model, let-7b-5p was confirmed to be differentially expressed both in peripheral blood and cerebral cortical tissue in the NE animal models when compared to control pups.

\section{Alteration in YAP expression of Hippo pathway in the rat neonatal encephalopathy models}

To understand the relationship between let-7b-5p and the Hippo pathway in the in vivo models, YAP expression was studied in the cerebral cortex of both NE animal models in comparison to the control pups (Fig. 7a) as for the cell cultures using co-staining between NeuN with YAP (neuronal, Fig. 7b) and YAP with Hoechst (nuclear, Fig. 7c).

In the cerebral cortex, there was a significant increase in the ratio of neuronal to nuclear YAP in NE animal models when compared to control pups (Fig. 7d). The neuronal/nuclear YAP ratio was significantly higher (mean +/- SEM) in hypoxic-ischaemic pups, ipsilateral side $(0.92+/-0.01, p<0.001)$ and contralateral side $(1.0+/-0.05, p<0.001)$, and in intrauterine inflammatory model pups $(0.95+/-0.01, p<0.001)$ when compared to control pups $(0.74+/-0.02)$, indicating a higher proportion of neuronal cytoplasmic YAP to nuclear YAP in the cerebral cortex of NE models (Fig. 7d). This neuronal/nuclear YAP ratio received contribution from the cytoplasmic YAP expression that was significantly higher (mean +/- SEM) in the hypoxic-ischaemic ipsilateral side $(40.5+/-3.7, p=0.002)$, hypoxic-ischaemic contralateral side $(39.5+/$ $3.4, p=0.003)$ and intrauterine inflammatory model pups $(38.3+/-2.3, p=0.001)$ when compared to control pups (Fig. 7e). The nuclear YAP staining was not significantly different in expression across both $\mathrm{NE}$ animal modes $(p=0.26)$ compared to control pups. (Fig. $7 f)$. These results further confirmed that activation of the Hippo pathway in the neurones of cerebral cortex in both NE models leads to apoptosis.

\section{Discussion}

While miRNAs have been noted to be involved in a number of pathophysiological processes in the central nervous system, our understanding of their role in neonates with moderate to severe NE is limited ${ }^{4}$. This study has used unbiased miRNA NGS to identify candidate miRNAs from DBS in neonates that may be associated with moderate to severe NE. Bioinformatic analysis yielded let-7b-5p as a potential candidate and miR-454-5p as an endogenous control miRNA. Furthermore, for the first time, we report the association of let-7b-5p with the apoptotic Hippo pathway in the context of NE, following validation of let$7 b-5 p$ in an independent cohort of neonates with NE. To enable the study of the cellular and molecular mechanism associated with let-7b-5p and the Hippo pathway in NE, in vitro and in vivo models of NE were used. In vitro, glucose-deprived neuronal cells mimicking metabolic stress in NE showed a decrease in let-7b-5p, an increase in cytoplasmic YAP expression and a reduction in nuclear YAP expression. In animal models of $\mathrm{NE}$, there was a significant decrease in let-7b-5p expression in peripheral blood of the rat intrauterine inflammation injury model and in the cerebral cortical neuronal cells of both the rat perinatal injury models. This was associated with a significant increase in neuronal cleaved caspase-3 
expression and cytoplasmic YAP expression in the cerebral cortex of both animal models, indicating neuronal apoptosis linked to activation of the Hippo pathway.

Whilst little is known about let-7b-5p in NE, let-7b has been observed to be expressed in the mammalian brain and has been studied in a number of neurological conditions such as ischaemic stroke ${ }^{18}$, glioma $^{19}$ and dementia ${ }^{20}$. Let-7b has also been shown to regulate neural stem cell proliferation and differentiation ${ }^{21,22}$. Additionally, extracellular let-7b may be a strong activator for Toll-like-Receptor signaling of the inflammatory pathway involved in adult neuronal cell death. ${ }^{23}$. In the present study, let$7 b-5 p$ was predominantly expressed in neuronal cells in both the NE animal models, with significant reduction in expression in the cerebral cortex of pups with hypoxic-ischaemic injury and intrauterine inflammation when compared to control pups.

Interestingly, we also noted a significant decrease in let-7b-5p expression in the peripheral blood of pups of the intrauterine inflammation model, but not the hypoxic-ischaemic injury model. One possible explanation for this discrepancy may be related to the time period from the insult given; the hypoxicischaemic pups were euthanised at $24 \mathrm{~h}$ post insult, while intrauterine inflammation pups had samples taken 9 days post insult, thus allowing more time for changes in brain miRNA to be observed peripherally. Given the limited knowledge about expression of let-7b-5p in NE, use of two different animal models with different injury patterns allowed us to explore the let-7b-5p expression at the cellular level. Cleaved caspase-3 positive apoptotic neuronal cell death was significantly higher in the cerebral cortex of both NE models when compared to control pups. This is in agreement with other studies showing activation of caspase-3 in neonatal hypoxic-ischaemic models both in rodents ${ }^{24,25}$ and piglets ${ }^{26}$. The corresponding reduction in let-7b-5p and increase in cleaved caspase-3 expression highlights the potential role of let-7b$5 p$ in the neuronal apoptotic cell death pathway ${ }^{27}$.

Let-7b regulation has been noted to involve a number of targets and pathways including vascular endothelial growth factor ${ }^{28}$, hypoxia inducible factor ${ }^{29}$ and neuroinflammation ${ }^{23,30}$, which are likely to be involved in the pathophysiology of NE. However, to date, let-7b-5p has not been studied in clinical or animal models of NE. Due to the presence of neuronal apoptosis in our models, we studied the role of let$7 b-5 p$ in the Hippo pathway, which was highlighted as one of the main apoptotic pathways through KEGG analysis.

The main function of the Hippo pathway depends on the translocation of YAP from the cytoplasm into the nucleus. Recently, a reduction in nuclear YAP protein levels has been correlated to reduced survival in Huntington's disease ${ }^{31}$. Although both the Hippo pathway and let-7b have been well studied independently in a number of oncological conditions, their role in NE remains to be explored in detail. In general, nuclear YAP is a potent growth promoter, and let-7b has a well-known tumour suppressor role ${ }^{32}$. We speculate that the interaction between nuclear YAP and let-7b-5p may be inverse in order to maintain a normal environment with adequate cell growth /differentiation through a complex feedback system 
involving a number of anti-apoptotic genes and possibly other miRNAs. In NE, the down regulation of let$7 b-5 p$ and the reduction in nuclear YAP could result in apoptotic neuronal cell death.

Currently, knowledge about specific interactions between the Hippo pathway and let-7b-5p is limited. A number of miRNAs have been noted to both positively and negatively regulate the Hippo pathway upstream $^{33}$. On the other hand, the Hippo pathway can also regulate let-7b either directly through Dicer expression ${ }^{34}$, indirectly through Lin28 which is influenced by phosphorylated Merlin, an upstream tumour suppressor in the Hippo pathway ${ }^{33,35}$ or by p72 binding to the microprocessor complex needed in the biogenesis of miRNAs ${ }^{36}$. Some of these mechanisms also provide an association between the Hippo pathway and biogenesis of other miRNAs, with or without involving let-7b expression based on cell contact signals ${ }^{34,36}$. There are complex feedback loops between various miRNAs and the Hippo pathway, effectively controlling the expression of nuclear YAP, which in turn regulates anti-apoptotic genes and thus controls cell growth and survival. These need to be explored further.

Our study has a number of strengths. We have used neonatal samples from well defined groups of babies with NE as a starting point to identify differentially expressed miRNAs through unbiased next generation sequencing and further validated the candidate miRNA in a larger independent cohort of neonates using RT-qPCR. We have demonstrated a novel mechanism for let-7b in NE acting through the Hippo-YAP-let-7b axis using both in vitro and in vivo models.

Studies using umbilical cord blood as a biosample source to identify potential early biomarkers to stratify neonates with NE have been reported ${ }^{37-39}$. However, using miRNA NGS, we have also shown that umbilical cord blood has a different miRNA profile compared to that of neonatal blood and thus is not suitable as a control biosample for neonatal blood samples from later time points. Finally, in this study we have validated our original technique of using DBS for RT-qPCR ${ }^{8,9}$ to additionally perform miRNA NGS analysis. This would improve the feasibility of performing future large-scale studies in the neonatal population.

The main limitations of our study are the relatively smaller number of samples in each subgroup for various grades of NE in the miRNA NGS study and the individual variations in the sample collection times for the sequential blood samples. This might explain the lack of clear identification of significant miRNAs differentially expressed between the groups of neonates with varying degrees of NE. While the number of samples used provided necessary power for statistical analysis, neonates with encephalopathy are known to have varying clinical presentation that evolves with time. Therefore, a larger sample size in the subgroups at precisely set time points would have been more beneficial. We tried to reduce the clinical variations by selecting the neonates in each sub group using clear categorisation as described in Supplementary Table 1. Additionally, as a novel study on mechanism of action of a miRNA, we used both peripheral blood and brain tissue of the animal models along with cell cultures to study the changes seen in NE at cellular level. Whilst this is a robust technique, the findings may not be fully transferable to human neonates due to known variation in expression of miRNAs in different species and tissues. 
However, of all miRNAs, let-7b is known to be an extremely well conserved miRNA across various species including humans and rodents.

In summary, through miRNA NGS analysis using dried blood spots of neonates with NE, and both in vitro and in vivo models of NE, this study highlights the potential importance of let-7b-5p and YAP in the Hippo pathway, in apoptotic neuronal cell death in NE. The complex interactions of let-7b-5p with its other targets and miRNAs warrant further investigation of its role in the pathophysiology of NE.

\section{Methods And Materials}

\section{Clinical Cohort}

Between Jan 2014 to Jan 2016, neonates > 36 weeks' gestation were recruited as part of the BIBiNS (Brain Injury Biomarkers in NewbornS) study from five UK neonatal units: The Royal London Hospital (Barts Health NHS Trust), Homerton University Hospital NHS Foundation Trust, Ashford and St Peter's Hospitals NHS Foundation Trust, University Hospital Southampton NHS Foundation Trust, and Norfolk and Norwich University Hospitals NHS Foundation Trust. The study was approved by a UK research ethics committee (REC ref:13/LO/1738). Neonates were recruited with written consent from parents.

All eligible neonates were categorised prospectively into three groups based on their clinical presentation. Group 1 included neonates with a clinical diagnosis of moderate to severe NE who fulfilled standard cooling criteria ${ }^{40,41}$ and received TH for $72 \mathrm{~h}$. Group 2 included neonates with mild NE admitted to the neonatal unit with perinatal acidosis but did not satisfy criteria for TH treatment. Group 3 were cord blood samples from normal pregnancies. Neonates in Group 1 treated with TH were further divided into 2 subgroups: those with cerebral MRI predictive of either favourable (Group 1A) or unfavourable outcome (Group 1B), based on a validated scoring system ${ }^{42}$ as previously described ${ }^{16}$.

\section{Samples Studied}

The neonates who underwent TH (Group 1) had samples collected at three time points: sample 1 (S1) obtained on day 1 after achieving target body temperature of $33.5^{\circ} \mathrm{C}$; sample 2 (S2) obtained between days 2-3 during cooling therapy and prior to commencing rewarming; and sample 3 (S3) obtained on day 5 after rewarming was complete. Group 2 neonates had a single sample collected within 48 hours of birth while Group 3 neonates had umbilical cord samples collected at birth. A drop of blood was collected at each sample point on an absorbent filter paper (Whatman 903 protein saver card) to form a DBS and stored in a polythene bag with a packet of desiccant at room temperature. Supplementary Fig. 1 shows the grouping of neonates and types of samples collected.

\section{MiRNA next-generation sequencing}

Based on the prediction of generating around 20 million reads per sample, a minimum sample size of 20 samples was estimated for miRNA NGS analysis. A cohort of 16 neonates was included, with four per 
subgroup selected on the basis of robust clinical grouping and MRI outcome to provide a total of 32 samples at the three time points described above. The clinical characteristics of these neonates are described in Supplementary Table 1. With $2 \times 6 \mathrm{~mm}$ DBS chad and previously published method ${ }^{9}$ MiRNA NGS was performed using Illumina Nextseq 500 high output run ( 75 cycles) kit ${ }^{43}$.

\section{Kyoto Encyclopedia of Genes and Genomes pathways analysis}

MiRNA pathway analysis was performed using mirPath V.3 to predict miRNA targets ${ }^{44}$. The Kyoto Encyclopedia of Genes and Genomes (KEGG) pathways analysis was performed for three different comparisons through Tarbase V7.0 using pathway union. This included: 1) mild NE versus moderate to severe NE with favourable outcome (Supplementary Fig. 2), 2) mild NE versus moderate to severe NE with unfavourable outcome (Supplementary Fig. 3), and 3) moderate to severe NE with favourable outcome versus moderate to severe NE with unfavourable outcome (Supplementary Fig. 4). Reverse Pathway analysis of identified pathways was also performed for confirmation of the selected signalling pathway.

\section{Validation using quantitative Reverse Transcription PCR}

The TaqMan microRNA assays for quantitative RT-qPCR was performed according to manufacturer's protocols as previously described ${ }^{9}$. The three potential candidate miRNAs: let-7b-5p (Assay ID: 000378); let-7b-3p (Assay ID: 002404); and miR-3200-3p (Assay ID: 241643), plus the endogenous control miRNA miR-454-5p (Assay ID: 001996) were obtained from our miRNA NGS study. Validation by RT-qPCR was performed on both the miRNA NGS cohort and another larger independent cohort.

\section{Cell cultures}

Primary mixed cell cultures (containing approx. 27\% neurones) from C57BL/6 mice pups at post-natal day 2 were carried out according to previously published work ${ }^{45}$. In order to study cell cultures under conditions of stress, glucose-deprivation was performed ${ }^{46}$. Cell cultures at a density of 6,000 cells per well in normal and glucose-deprived conditions were studied using combined FISH - IHC to identify the microRNA let-7b-5p expression in neurons using mouse anti-NeuN (1:500, MAB377, Merck Millipore $)^{47}$. The cell cultures were double immunostained for YAP (rabbit anti-YAP, 1:100, 14074S, Cell Signaling Technology) and NeuN, with nuclear Hoechst staining as previously described ${ }^{48}$.

\section{Rodent models of perinatal brain injury}

All animal research was performed under the EU adopted Directive 2010/63/EU and reviewed by the local Comité National de Réflexion Ethique sur l'Expérimentation animale. The in vivo animal experiments for two established perinatal brain injury models for NE and control pups were carried out on time-pregnant Wistar rats purchased from CERJ (Le Genest, France). In the hypoxic-ischaemic model, pups of either sex at post-natal day 7 were anaesthetised using isoflurane and a unilateral common carotid artery ligation followed by hypoxia in a chamber was carried out as described before ${ }^{49}$. After $24 \mathrm{~h}$, the pups were deeply anaesthetised, blood was collected onto absorbent filter paper as DBS with brains were dissected and stored in $20 \%$ sucrose at $4^{\circ} \mathrm{C}$ until further processed. The intrauterine inflammation model involved an intraperitoneal injection of $300 \mu \mathrm{g} / \mathrm{kg}$ of lipopolysaccharide (E. coli, serotype 055:B5), to a pregnant rat at 
20 days of gestation to produce inflammation, as previously described ${ }^{50}$. The blood and brain tissues were collected from pups on post-natal day 8 as described earlier. The control animals were from a group of naïve rat pups that underwent normal pregnancy conditions and were sacrificed on post-natal day 8 for blood and brain collection similar to experimental groups.

Ten $\mu \mathrm{m}$ brain sections between Bregma co-ordinates -2.20 to $-0.20 \mathrm{~mm}$ containing the cerebral cortex, as shown in the postnatal rat brain atlas ${ }^{51}$ were used in this study. The identification of the cell type that expressed let-7b-5p in the brain of rat animal models using mouse anti-NeuN (1:500, MAB377) for neurones was carried out using FISH and IHC, respectively. A similar protocol was used for double IHC using tyramide amplification for studying neuronal cell death in the animal models using rabbit anticleaved caspase 3 (1:100, Cat No. 9664, Cell Signaling Technology), and for expression of YAP in neurones using rabbit anti-YAP (1:200, Cat No. 14074S, Cell Signaling Technology). Each animal model $(n=3-6)$ had 3-4 brain sections studied and imaged. In the hypoxic-ischaemic model, the injured side was defined as the ipsilateral side due to the more focal nature of the insult. In the intrauterine inflammation model, both left and right sides were combined together for analysis as the brain was affected globally by the endotoxin. Similarly, the uninjured control brain was analysed with combination of data from both the left and right side. Images were analysed using Image J (1.51v) with a customised script.

\section{Statistics}

Bioinformatics analysis for miRNA NGS data was performed using Partek® Genomics Suite® software ${ }^{52}$. Heat maps and PCA were performed to identify differentially expressed miRNAs between the groups and at various time points. Normfinder was used to identify miRNAs with best stability factor, to be suitable endogenous control miRNAs ${ }^{53}$. Relative quantification of the candidate miRNAs were performed using $2^{-\Delta \Delta C t}$ method ${ }^{54}$. Hierarchical clustering was performed using R program ${ }^{55}$. All other data analysis was performed using GraphPad Prism 8. All statistical tests were 2-tailed with a significance level set with an alpha of 0.05 . For continuous variables, statistical significance between three patient groups was compared using one-way ANOVA with Dunnett's post hoc test when comparing with the control group. For categorical variables, Pearson's chi-square and Fisher's Exact tests were used. For two group comparisons, the Mann-Whitney $U$ test was used for non-parametric continuous variables while the unpaired t-test was used for parametric data.

\section{Abbreviations}

DBS, Dried blood spot; DMEM, Dulbecco's modified Eagle medium; FDR, False detection ratio, FISH, Fluorescent in-situ hybridisation; IHC, Immunohistochemistry; miRNA, microRNA; NE, Neonatal encephalopathy; NGS, Next-generation sequencing; RT-qPCR, Reverse Transcription and quantitative Polymerase Chain Reaction; S, Sample; TAZ, transcriptional coactivator with PDZ-binding motif; TEAD, TEA domain; TH, Therapeutic hypothermia; YAP, Yes Associated Protein. 


\section{Declarations}

\section{Acknowledgements}

We are grateful to the neonates and the families who participated in BIBiNs study and thank the medical and nursing staff on all the neonatal units for their support. We would like to thank Ms Nicola OpenshawLawrence, Nicky Holland, Vicky Payne and Karen Few for assistance with patient recruitment and data coordination at Royal London, Ashford \& St Peter's, Southampton and Norwich hospitals respectively. We are also grateful for the work done by Dr Jane Evanson and Dr Olga Kapellou in reporting the MRI brain scans. We are very grateful to Barts Charity, London, UK for funding support for the study.

\section{Author Contributions}

VP, PY, PG, and DS contributed to the conception and design of the study; VP, PY, RI, MM, PC, EW, CM, LS, $A B, P C$ and $E C$ contributed to acquisition and analysis of the data; and VP, AMT, PG, PY and DS contributed to drafting the text and preparing the figures.

\section{Competing Interests statement}

All authors report no competing interest.

\section{References}

1 Lee, A. C. C. et al. Intrapartum-related neonatal encephalopathy incidence and impairment at regional and global levels for 2010 with trends from 1990. Pediatr. Res. 74 Suppl 1, 50-72, doi:10.1038/pr.2013.206 (2013).

2 Edwards, A. D. et al. Neurological outcomes at 18 months of age after moderate hypothermia for perinatal hypoxic ischaemic encephalopathy: synthesis and meta-analysis of trial data. The BMJ $\mathbf{3 4 0}$, c363, doi:10.1136/bmj.c363 (2010).

3 Jacobs, S. E. et al. Cooling for newborns with hypoxic ischaemic encephalopathy. Cochrane Database Syst. Rev., Cd003311, doi:10.1002/14651858.CD003311.pub3 (2013).

4 Ponnusamy, V. \& Yip, P. The role of microRNAs in newborn brain development and hypoxic ischaemic encephalopathy. Neuropharmacology 149, 55-65, doi:https://doi.org/10.1016/j.neuropharm.2018.11.041 (2019).

5 Cheng, G. Circulating miRNAs: Roles in cancer diagnosis, prognosis and therapy. Adv Drug Deliv Rev 81C, 75-93, doi:10.1016/j.addr.2014.09.001 (2015).

6 Olson, E. N. MicroRNAs as therapeutic targets and biomarkers of cardiovascular disease. Sci. Transl. Med. 6, 239ps233, doi:10.1126/scitranslmed.3009008 (2014). 
7 Dumache, R. et al. Use of miRNAs as biomarkers in sepsis. Anal. Cell. Pathol. (Amst.) 2015, 186716, doi:10.1155/2015/186716 (2015).

8 Shi $\mathrm{J}$ et al. Significance of serum miRNA-21 expression in neonates with hypoxic-ischemic encephalopathy. Pract. Prev. Med. 25, 655-658, doi:10.3969/j.issn.1006-3110.2018.06.005 (2018).

9 Ponnusamy, V. et al. A study of microRNAs from dried blood spots in newborns after perinatal asphyxia: a simple and feasible biosampling method. Pediatr. Res. 79, 799-805, doi:10.1038/pr.2015.276 (2016).

10 Wang, Z., Liu, Y., Shao, M., Wang, D. \& Zhang, Y. Combined prediction of miR-210 and miR-374a for severity and prognosis of hypoxic-ischemic encephalopathy. Brain Behav 8, e00835, doi:10.1002/brb3.835 (2018).

11 O'Sullivan, M. P. et al. Validation of Altered Umbilical Cord Blood MicroRNA Expression in Neonatal Hypoxic-Ischemic Encephalopathy. JAMA neurology 76, 333-341, doi:10.1001/jamaneurol.2018.4182 (2019).

12 Harvey, K. F., Pfleger, C. M. \& Hariharan, I. K. The Drosophila Mst ortholog, hippo, restricts growth and cell proliferation and promotes apoptosis. Cel/ 114, 457-467, doi:10.1016/s0092-8674(03)00557-9 (2003).

13 Wu, S., Huang, J., Dong, J. \& Pan, D. hippo encodes a Ste-20 family protein kinase that restricts cell proliferation and promotes apoptosis in conjunction with salvador and warts. Cel/ 114, 445-456, doi:10.1016/s0092-8674(03)00549-x (2003).

14 Holden, J. K. \& Cunningham, C. N. Targeting the Hippo Pathway and Cancer through the TEAD Family of Transcription Factors. Cancers (Basel) 10, doi:10.3390/cancers10030081 (2018).

15 Wu, S., Liu, Y., Zheng, Y., Dong, J. \& Pan, D. The TEAD/TEF family protein Scalloped mediates transcriptional output of the Hippo growth-regulatory pathway. Dev Cell 14, 388-398, doi:10.1016/j.devcel.2008.01.007 (2008).

16 Tharmapoopathy, P. et al. In clinical practice, cerebral MRI in newborns is highly predictive of neurodevelopmental outcome after therapeutic hypothermia. Eur. J. Paediatr. Neurol. 25, 127-133, doi:10.1016/j.ejpn.2019.12.018 (2020).

17 Shreberk-Shaked, M. \& Oren, M. New insights into YAP/TAZ nucleo-cytoplasmic shuttling: new cancer therapeutic opportunities? Mol. Oncol. 13, 1335-1341, doi:10.1002/1878-0261.12498 (2019).

18 Long, G. et al. Circulating miR-30a, miR-126 and let-7b as biomarker for ischemic stroke in humans. BMC Neurol 13, 178, doi:10.1186/1471-2377-13-178 (2013). 
19 Zhang, W. et al. Decreased let-7b is associated with poor prognosis in glioma. Medicine (Baltimore) 98, e15784, doi:10.1097/md.0000000000015784 (2019).

20 Liu, Y., He, X., Li, Y. \& Wang, T. Cerebrospinal fluid CD4+ T lymphocyte-derived miRNA-let-7b can enhances the diagnostic performance of Alzheimer's disease biomarkers. Biochem Biophys Res Commun 495, 1144-1150, doi:10.1016/j.bbrc.2017.11.122 (2018).

$21 \mathrm{Ni}, \mathrm{N}$. et al. Effects of let-7b and TLX on the proliferation and differentiation of retinal progenitor cells in vitro. Sci. Rep. 4, 6671, doi:10.1038/srep06671 (2014).

22 Zhao, C. et al. MicroRNA let-7b regulates neural stem cell proliferation and differentiation by targeting nuclear receptor TLX signaling. Proc. Natl. Acad. Sci. U. S. A. 107, 1876-1881, doi:10.1073/pnas.0908750107 (2010).

23 Lehmann, S. M. et al. An unconventional role for miRNA: let-7 activates Toll-like receptor 7 and causes neurodegeneration. Nat Neurosci 15, 827-835, doi:10.1038/nn.3113 (2012).

24 Northington, F. J., Ferriero, D. M., Flock, D. L. \& Martin, L. J. Delayed neurodegeneration in neonatal rat thalamus after hypoxia-ischemia is apoptosis. J. Neurosci. 21, 1931-1938, doi:10.1523/jneurosci.2106-01931.2001 (2001).

25 Blomgren, K. et al. Synergistic activation of caspase-3 by m-calpain after neonatal hypoxiaischemia: a mechanism of "pathological apoptosis"? J. Biol. Chem. 276, 10191-10198, doi:10.1074/jbc.M007807200 (2001).

26 Delivoria-Papadopoulos, M., Ashraf, Q. M., Ara, J. \& Mishra, O. P. Nuclear mechanisms of hypoxic cerebral injury in the newborn: the role of caspases. Semin. Perinatol. 32, 334-343, doi:10.1053/j.semperi.2008.07.002 (2008).

27 Ham, 0. et al. let-7b suppresses apoptosis and autophagy of human mesenchymal stem cells transplanted into ischemia/reperfusion injured heart 7by targeting caspase-3. Stem Cell. Res. Ther. 6, 147, doi:10.1186/s13287-015-0134-x (2015).

28 Hua, Z. et al. MiRNA-directed regulation of VEGF and other angiogenic factors under hypoxia. PLoS One 1, e116, doi:10.1371/journal.pone.0000116 (2006).

29 Zhang, R. et al. MiRNA let-7b promotes the development of hypoxic pulmonary hypertension by targeting ACE2. Am J Physiol Lung Cell Mol Physio/ 316, L547-I557, doi:10.1152/ajplung.00387.2018 (2019).

30 Slota, J. A. \& Booth, S. A. MicroRNAs in Neuroinflammation: Implications in Disease Pathogenesis, Biomarker Discovery and Therapeutic Applications. Non-coding RNA 5, 35, doi:10.3390/ncrna 5020035 (2019). 
31 Mueller, K. A. et al. Hippo Signaling Pathway Dysregulation in Human Huntington's Disease Brain and Neuronal Stem Cells. Sci Rep 8, 11355, doi:10.1038/s41598-018-29319-4 (2018).

32 Liu, C. et al. Distinct microRNA expression profiles in prostate cancer stem/progenitor cells and tumor-suppressive functions of let-7. Cancer Res. 72, 3393-3404, doi:10.1158/0008-5472.CAN-11-3864 (2012).

$33 \mathrm{Li}, \mathrm{N} ., \mathrm{Xie}, \mathrm{C}$. \& Lu, N. Crosstalk between Hippo signalling and miRNAs in tumour progression. Febs j 284, 1045-1055, doi:10.1111/febs.13985 (2017).

34 Chaulk, S. G., Lattanzi, V. J., Hiemer, S. E., Fahlman, R. P. \& Varelas, X. The Hippo pathway effectors TAZ/YAP regulate dicer expression and microRNA biogenesis through Let-7. J Biol Chem 289, 1886-1891, doi:10.1074/jbc.C113.529362 (2014).

35 Zhang, N. et al. The Merlin/NF2 tumor suppressor functions through the YAP oncoprotein to regulate tissue homeostasis in mammals. Dev. Cell 19, 27-38, doi:10.1016/j.devcel.2010.06.015 (2010).

36 Mori, M. et al. Hippo signaling regulates microprocessor and links cell-density-dependent miRNA biogenesis to cancer. Cell 156, 893-906, doi:10.1016/j.cell.2013.12.043 (2014).

37 Walsh, B. H. et al. The metabolomic profile of umbilical cord blood in neonatal hypoxic ischaemic encephalopathy. PLoS One 7, e50520-e50520, doi:10.1371/journal.pone.0050520 (2012).

38 Douglas-Escobar, M. V. et al. UCH-L1 and GFAP Serum Levels in Neonates with Hypoxic-Ischemic Encephalopathy: A Single Center Pilot Study. Front. Neurol. 5, 273, doi:10.3389/fneur.2014.00273 (2014).

39 Van Anh, T. N., Hao, T. K., Chi, N. T. D. \& Son, N. H. Predictions of Hypoxic-Ischemic Encephalopathy by Umbilical Cord Blood Lactate in Newborns with Birth Asphyxia. Open access Macedonian journal of medical sciences 7, 3564-3567, doi:10.3889/oamjms.2019.581 (2019).

40 Azzopardi, D. et al. The TOBY Study. Whole body hypothermia for the treatment of perinatal asphyxial encephalopathy: a randomised controlled trial. BMC Pediatr. 8, 17, doi:10.1186/1471-2431-8-17 (2008).

41 NPEU. The UK TOBY Cooling Register Clinician's Handbook, <https://npeu.ox.ac.uk/downloads/files/tobyregister/Register-Clinicans-Handbook1-v4-07-06-10.pdf> (2010).

42 Rutherford, M. et al. Assessment of brain tissue injury after moderate hypothermia in neonates with hypoxic-ischaemic encephalopathy: a nested substudy of a randomised controlled trial. Lancet Neurol. 9, 39-45, doi:10.1016/S1474-4422(09)70295-9 (2010).

43 Hayashi, H. et al. Preclinical study of DNA vaccines targeting SARS-CoV-2. bioRxiv, 2020.2010.2021.347799, doi:10.1101/2020.10.21.347799 (2020). 
44 Vlachos, I. S. et al. DIANA-miRPath v3.0: deciphering microRNA function with experimental support. Nucleic Acids Res. 43, W460-W466, doi:10.1093/nar/gkv403 (2015).

45 O'Meara, R. W., Ryan, S. D., Colognato, H. \& Kothary, R. Derivation of enriched oligodendrocyte cultures and oligodendrocyte/neuron myelinating co-cultures from post-natal murine tissues. $J$ Vis Exp, doi:10.3791/3324 (2011).

46 Monyer, H., Goldberg, M. P. \& Choi, D. W. Glucose deprivation neuronal injury in cortical culture. Brain Res. 483, 347-354, doi:https://doi.org/10.1016/0006-8993(89)90179-0 (1989).

47 Liu, Z. H. et al. A Single Bolus of Docosahexaenoic Acid Promotes Neuroplastic Changes in the Innervation of Spinal Cord Interneurons and Motor Neurons and Improves Functional Recovery after Spinal Cord Injury. J. Neurosci. 35, 12733-12752, doi:10.1523/jneurosci.0605-15.2015 (2015).

48 Yip, P., Wong, L.-F., Sears, T. A., Yáñez-Muñoz, R. J. \& McMahon, S. B. Cortical Overexpression of Neuronal Calcium Sensor-1 Induces Functional Plasticity in Spinal Cord Following Unilateral Pyramidal Tract Injury in Rat. PLoS Biol. 8, e1000399, doi:10.1371/journal.pbio.1000399 (2010).

49 Koning, G. et al. Magnesium induces preconditioning of the neonatal brain via profound mitochondrial protection. Journal of cerebral blood flow and metabolism : official journal of the International Society of Cerebral Blood Flow and Metabolism 39, 1038-1055, doi:10.1177/0271678X17746132 (2019).

50 Rousset, C. I. et al. Maternal exposure to lipopolysaccharide leads to transient motor dysfunction in neonatal rats. Dev Neurosci 35, 172-181, doi:10.1159/000346579 (2013).

51 Khazipov, R. et al. Atlas of the Postnatal Rat Brain in Stereotaxic Coordinates. Front. Neuroanat. 9, doi:10.3389/fnana.2015.00161 (2015).

52 Partek® Genomics Suite® v. 7 (Partek inc, St. Louis, 2018).

53 Andersen, C. L., Jensen, J. L. \& Ørntoft, T. F. Normalization of Real-Time Quantitative Reverse Transcription-PCR Data: A Model-Based Variance Estimation Approach to Identify Genes Suited for Normalization, Applied to Bladder and Colon Cancer Data Sets. Cancer Res. 64, 5245-5250, doi:10.1158/0008-5472.Can-04-0496 (2004).

54 Livak, K. J. \& Schmittgen, T. D. Analysis of Relative Gene Expression Data Using Real-Time Quantitative PCR and the 2- $\Delta \Delta C T$ Method. Methods 25, 402-408, doi:https://doi.org/10.1006/meth.2001.1262 (2001).

55 Team, R. C. R: A language and environment for statistical computing. . R Foundation for Statistical Computing, Vienna, Austria. (2013). 


\section{Table}

Table 1. Clinical characteristics of patient samples analysed by RT-qPCR for the validation cohort

Values are median (IQR) unless indicated otherwise; N/A, Not Applicable; $*, * *, * * *$ denote significant $\mathrm{p}<0.05, \mathrm{p}<0.01, \mathrm{p}<0.001$, respectively. $\mathrm{NE}$, neonatal encephalopathy; $\mathrm{TH}$, therapeutic hypothermia; DBS, dried blood spot.

\begin{tabular}{|c|c|c|c|c|}
\hline $\begin{array}{l}\text { Perinatal } \\
\text { characteristics }\end{array}$ & $\begin{array}{l}\text { Moderate to severe } \\
\text { NE with TH and } \\
\text { favourable MRI } \\
\text { outcome } \\
\text { (Group 1A) } \\
\mathrm{n}=15\end{array}$ & $\begin{array}{l}\text { Moderate to severe NE } \\
\text { with TH and unfavourable } \\
\text { MRI outcome (Group 1B) } \\
\mathrm{n}=15\end{array}$ & $\begin{array}{l}\text { Mild } \\
\text { NE } \\
\text { without } \\
\text { TH } \\
\text { (Group } \\
\text { 2) } \\
\text { n=15 }\end{array}$ & $P$ value \\
\hline $\begin{array}{l}\text { Gestational age, } \\
\text { (completed weeks + } \\
\text { days) }\end{array}$ & $\begin{array}{l}40+4 \\
(39+3 \text { to } 41+4)\end{array}$ & $\begin{array}{l}40+2 \\
(38+4 \text { to } 41)\end{array}$ & $\begin{array}{l}40+2 \\
(39+3 \\
\text { to } \\
41+4)\end{array}$ & 0.430 \\
\hline Male sex, n (\%) & $10(67 \%)$ & $12(80 \%)$ & $\begin{array}{l}6 \\
(40 \%)\end{array}$ & 0.071 \\
\hline Birth weight (g) & $\begin{array}{l}3700 \\
(3318-3905)\end{array}$ & \begin{tabular}{|l}
3240 \\
$(2745-3685)$
\end{tabular} & $\begin{array}{l}3520 \\
(3145- \\
3972) \\
\end{array}$ & 0.101 \\
\hline $\begin{array}{l}\text { Apgar score at } 10 \\
\text { min }\end{array}$ & $5(4-7)$ & $4(4-6)$ & $\begin{array}{l}9(8 \\
-10)\end{array}$ & $<0.001^{* * *}$ \\
\hline Worst $\mathrm{pH}$ within $1 \mathrm{~h}$ & $6.88(6.77-6.95)$ & $6.87(6.64-7.00)$ & $\mathrm{N} / \mathrm{A}$ & 0.923 \\
\hline $\begin{array}{l}\text { Worst base deficit } \\
\text { within } 1 \mathrm{~h}\end{array}$ & $\begin{array}{l}-17.45 \\
(-14.12 \text { to }-20.85)\end{array}$ & $\begin{array}{l}-19.3 \\
(-17 \text { to }-22.9)\end{array}$ & $\mathrm{N} / \mathrm{A}$ & 0.381 \\
\hline $\begin{array}{l}\text { Need for respiratory } \\
\text { support at } 10 \mathrm{~min}, \\
\mathrm{n}(\%)\end{array}$ & $12(80 \%)$ & $13(87 \%)$ & $\begin{array}{l}4 \\
(27 \%)\end{array}$ & $0.001 * *$ \\
\hline $\begin{array}{l}\text { Need for chest } \\
\text { compressions, } \mathrm{n}(\%)\end{array}$ & $2(13 \%)$ & $6(40 \%)$ & $1(7 \%)$ & $0.038 *$ \\
\hline $\begin{array}{l}\text { Antenatal sentinel } \\
\text { event present, n (\%) }\end{array}$ & $3(20 \%)$ & $1(7 \%)$ & $\mathrm{N} / \mathrm{A}$ & 0.598 \\
\hline $\begin{array}{l}\text { Normal } \\
\text { neurodevelopmental } \\
\text { outcome, n (\%) }\end{array}$ & $15(100 \%)$ & $1(7 \%)$ & $\mathrm{N} / \mathrm{A}$ & $<0.001 * * *$ \\
\hline $\begin{array}{l}\text { Age at DBS sample } \\
\text { S1 (decimal hours) }\end{array}$ & $\begin{array}{l}14.8 \\
(10.8-21.1)\end{array}$ & $\begin{array}{l}18.1 \\
(12.4-21.1)\end{array}$ & $\begin{array}{l}22.8 \\
(14.9- \\
32) \\
\end{array}$ & 0.07 \\
\hline $\begin{array}{l}\text { Age at DBS sample } \\
\text { S2 (decimal hours) }\end{array}$ & $\begin{array}{l}60.2 \\
(51.1-67.0) \\
\end{array}$ & $\begin{array}{l}54.6 \\
(51.1-61.4) \\
\end{array}$ & $\mathrm{N} / \mathrm{A}$ & 0.907 \\
\hline $\begin{array}{l}\text { Age at DBS sample } \\
\text { S3 (decimal hours) }\end{array}$ & $\begin{array}{l}97.8 \\
(88.9-104.1)\end{array}$ & $\begin{array}{l}95.4 \\
(87.0-100.4)\end{array}$ & $\mathrm{N} / \mathrm{A}$ & 0.643 \\
\hline
\end{tabular}


a

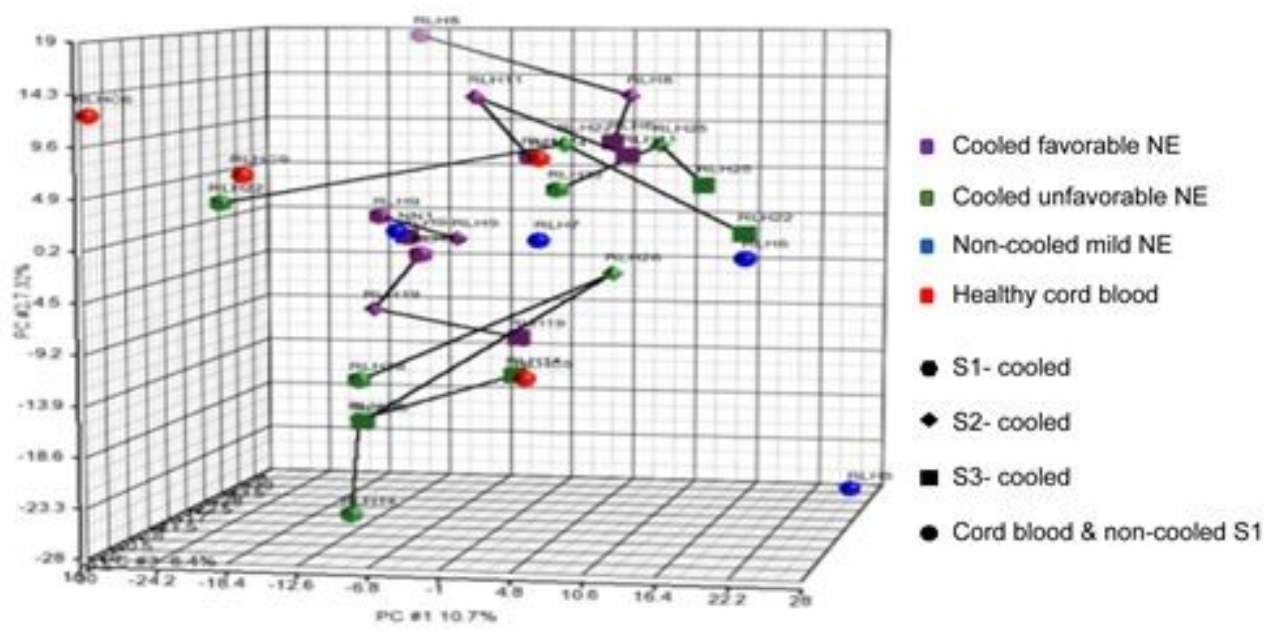

b
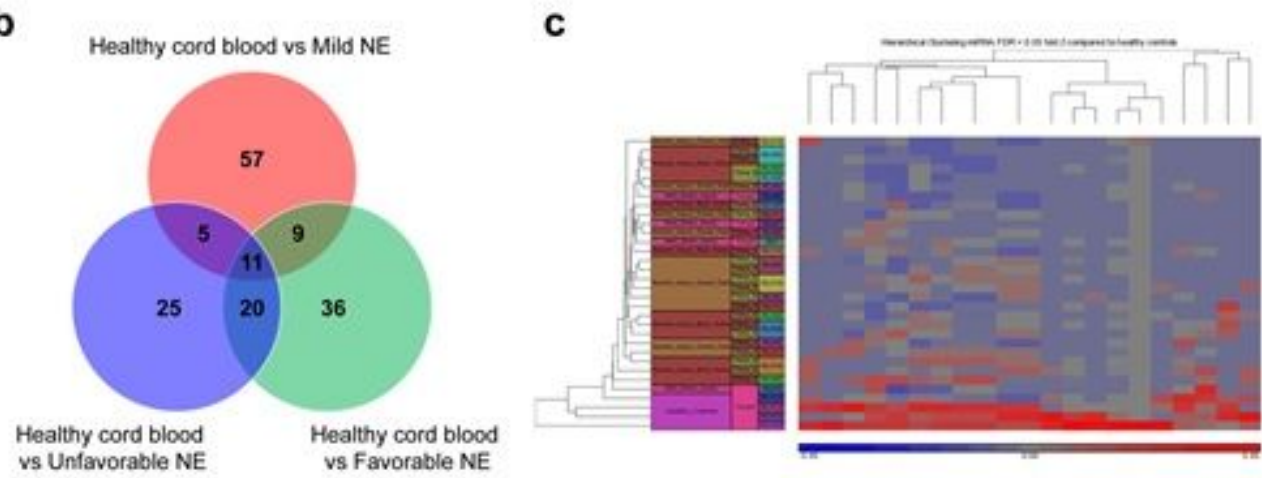

d

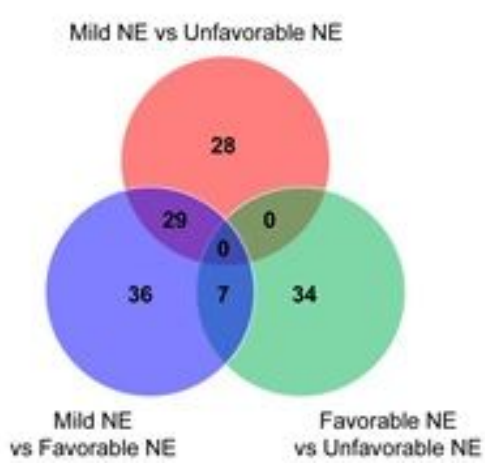

e
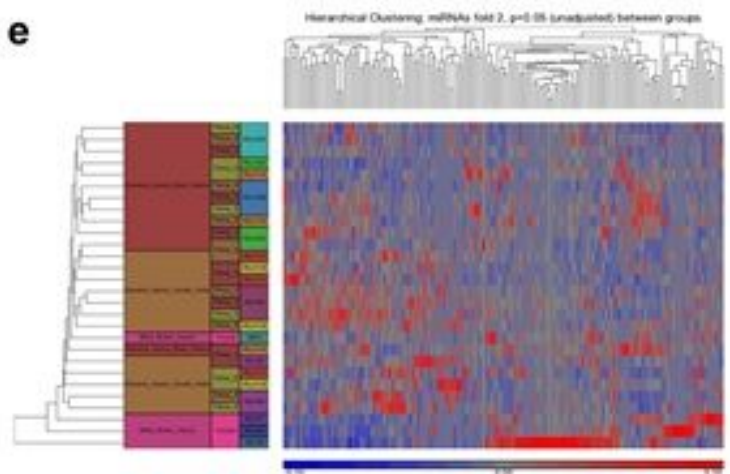

\section{Figure 1}

Identification of candidate miRNA through miRNA next generation sequencing. a PCA plot of all neonates examined with miRNA NGS according to their groups (Group 1A: moderate to severe NE with TH and favourable outcome (purple hexagon; S1, purple diamond; S2, purple square; S3), Group 1B: moderate to severe NE with TH and unfavourable outcome (green hexagon; S1, green diamond; S2, green square; S3), Group 2: mild NE without TH (blue circle), and Group 3: umbilical cord blood of healthy neonates (red circles). b Venn diagram showing the number of significant differential expression of miRNA between all three groups with umbilical cord blood. c Heatmap of all neonates in miRNA NGS with 17 significant miRNAs. $d$ Venn diagram showing the differential expression of miRNA between all three groups excluding umbilical cord blood. e Heatmap of all neonates in miRNA NGS excluding umbilical cord blood showing no significant miRNAs. 


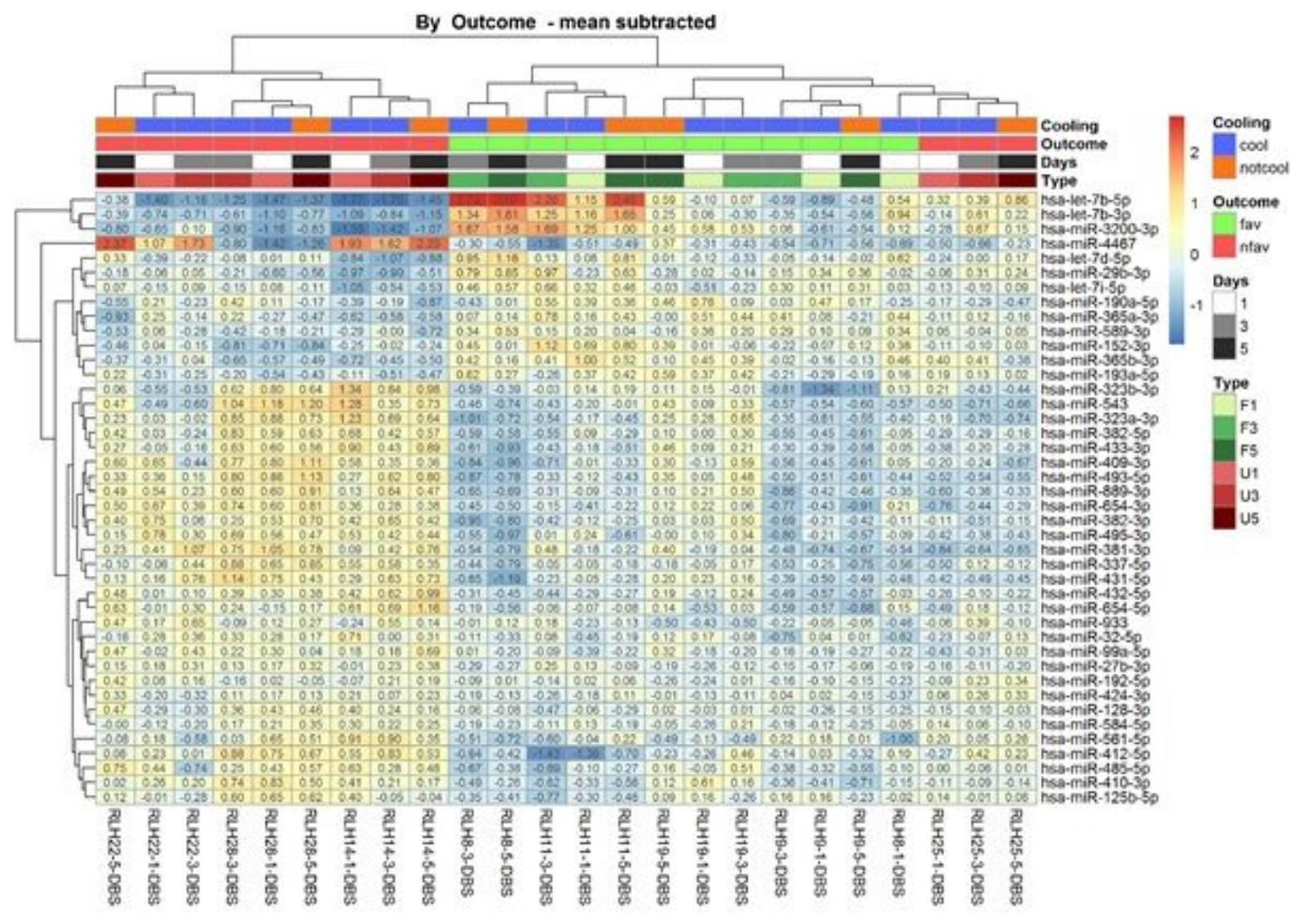

Figure 2

Hierarchical clustering analysis of all the differentially expressed miRNAs by outcome. Hierarchical clustering analysis using the R program showing let-7b in the forms of $3 p$ and $5 p$ strands to be the most significantly changed miRNA in the moderate to severe NE neonates with TH treatment based on outcome. Days 1, 3 and 5 correspond to samples S1, S2 and S3 respectively. F1, F3 and F5 represent favourable outcome on days 1, 3 and 5 respectively; while U1, U3 and U5 represent unfavourable outcome on days 1,3 and 5 respectively. 

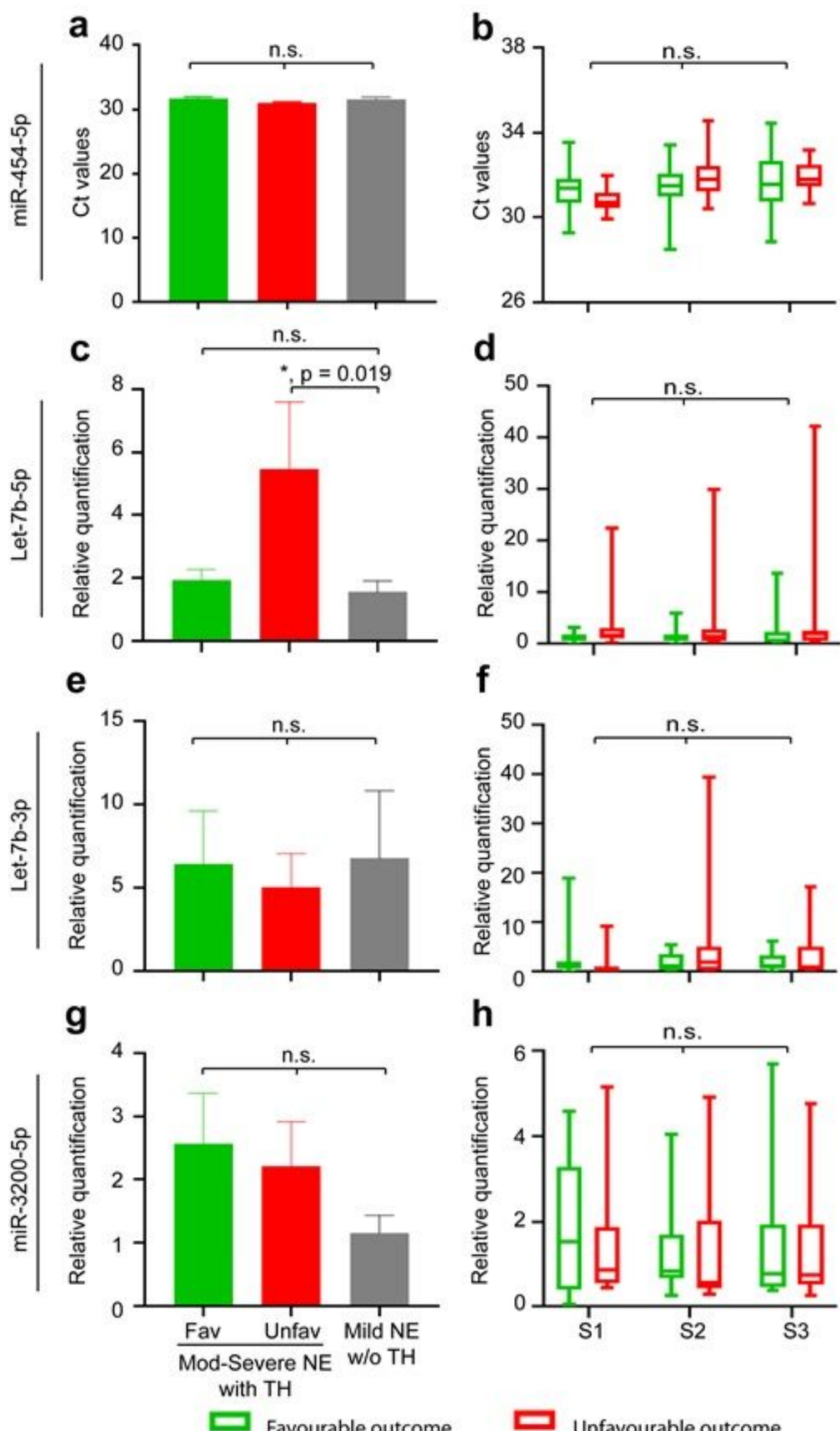

Favourable outcome

Unfavourable outcome

Figure 3

Expression of let-7b-3p and let-7b-5p in neonates with neonatal encephalopathy. $a, c, e$ and $g$ show the expression of miR-454-5p, let-7b-5p, let-7b-3p and miR-3200-5p in all groups at S1 samples. Similarly graphs $b, d, f$ and $h$ show the temporal expression of miR-454-5p, let-7b-5p, let-7b-3p and miR-3200-5p at various time points (S1, S2 and S3) in neonates with moderate to severe NE with TH and favourable and unfavourable outcome. Relative quantification denotes $2-\Delta \Delta C$ t of the candidate miRNAs in comparison 
to endogenous control miRNA, miR-454-5p. ns, not significant; * denote $p<0.05$ using Kruskal-Wallis test for non-parametric data. Each group consists of $n=15$ neonates. NE, neonatal encephalopathy; $T H$, therapeutic hypothermia; Fav, favourable outcome; Unfav, unfavourable outcome.
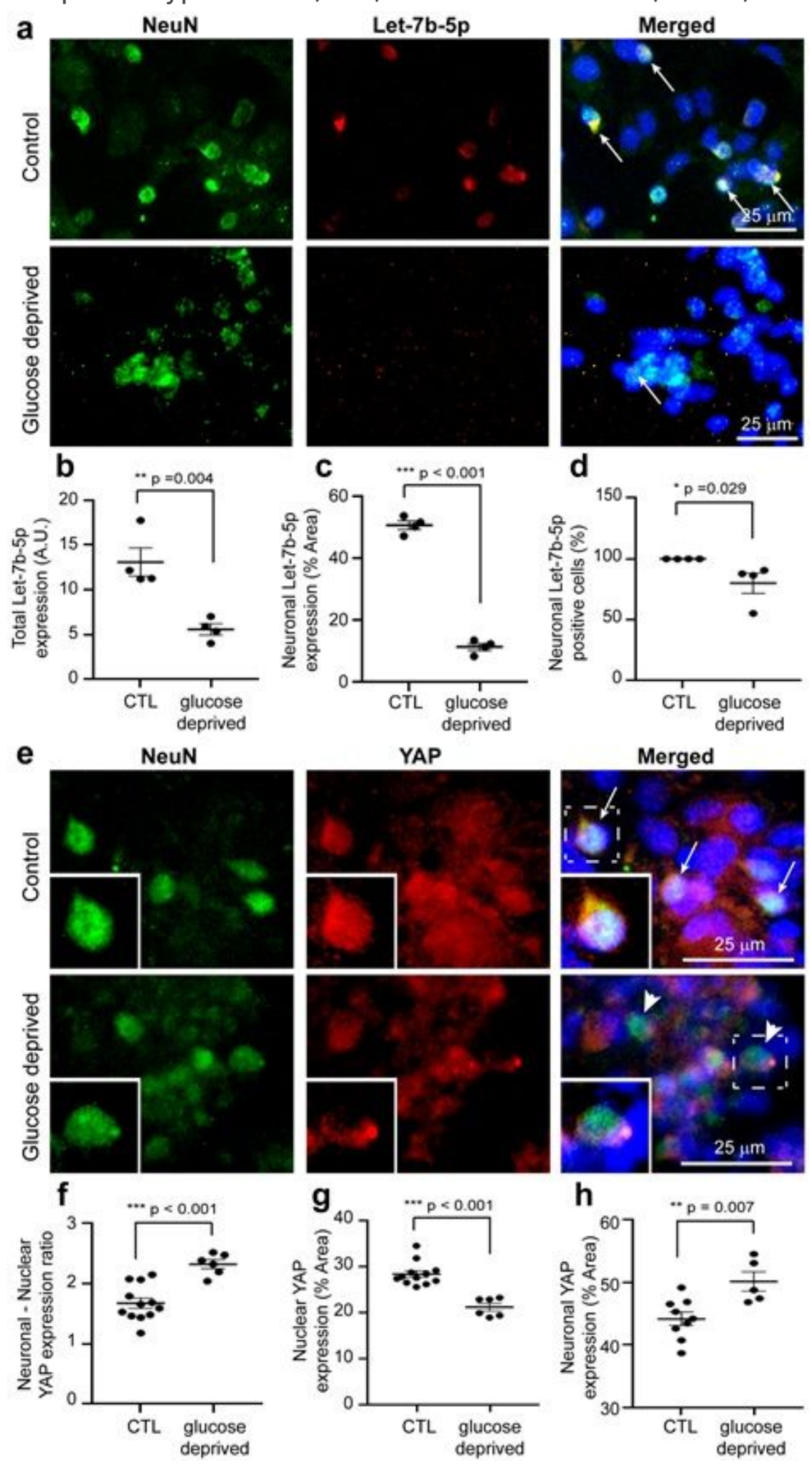

Figure 4

Neuronal expression of let-7b-5p and YAP in normal and glucose-deprived neuronal cells in vitro. a Images of NeuN positive neuronal cells (green) with let-7b-5p (red) and co-expression is shown (yellow) in 
the control and glucose-deprived group. Arrows indicate co-expression. b-d Graphs showing significant decrease in total let-7b-5p (b), neuronal let-7b-5p (c) and the percentage of neuronal let-7b-5p (d) after glucose-derivation compared to the control group. e Images of NeuN positive neuronal cells (green) with YAP (red) and co-expression is shown (yellow) in the control (arrows indicate cytoplasm expression) and glucose-deprived group (arrowheads indicate nuclear expression). f-h Graphs showing significant increase in neuronal/nuclear ratio (f), a significant decrease in nuclear YAP expression (g) and a significant increase in neuronal YAP expression $(h)$ after glucose-derivation compared to the control group. All values are mean with SEM. $n=4-12 .{ }^{*}, * \star$, $* \star \star$ denote significant $p<0.05, p<0.01, p<0.001$, respectively using unpaired t test. Scale bar $=25 \mathrm{um}$. CTL, control. 

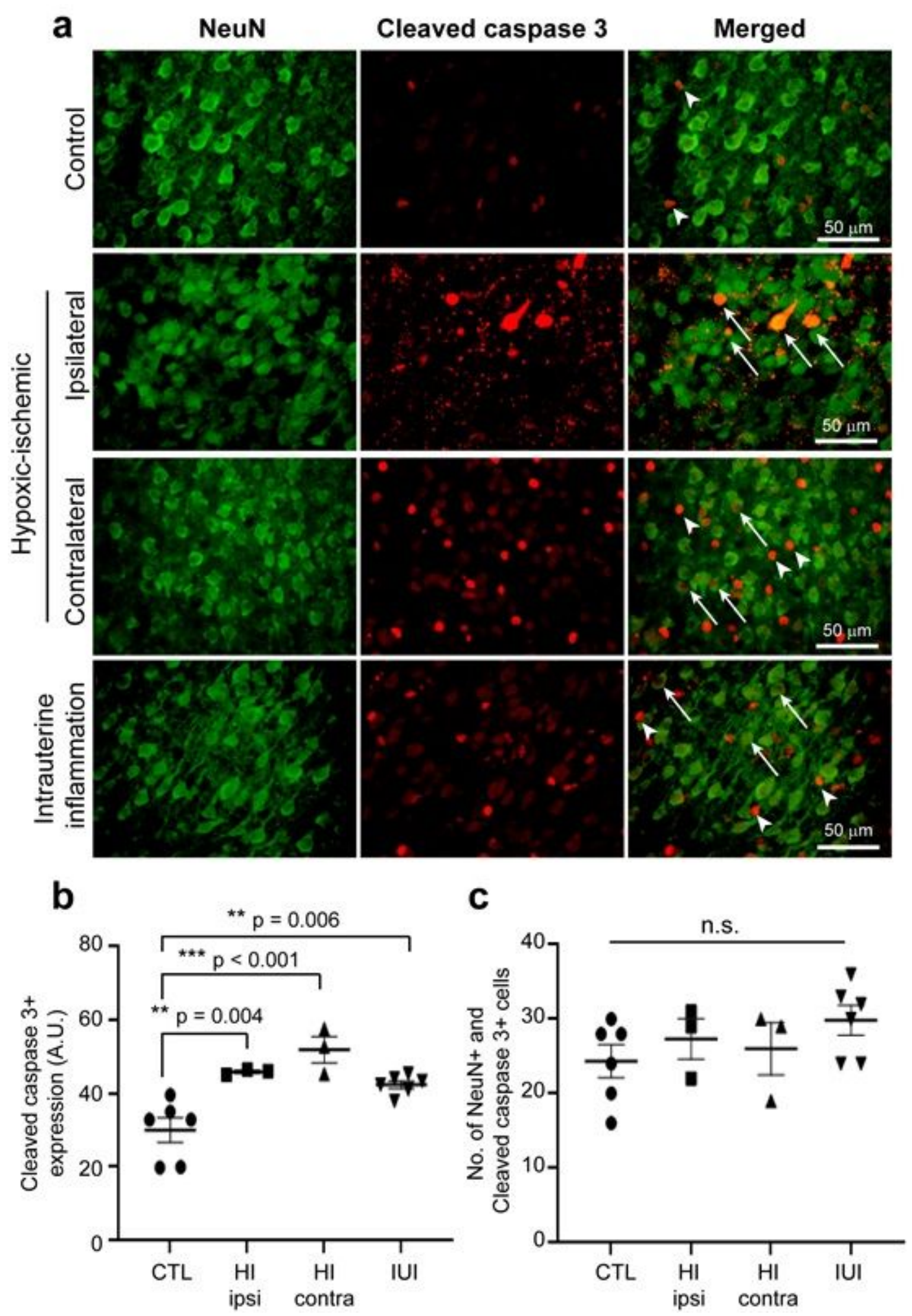

\section{Figure 5}

Neuronal apoptosis in the cerebral cortex of the rat neonatal encephalopathy models. a Images of neuronal cells (NeuN positive, green) that can undergo apoptosis (cleaved caspase-3 positive, red) and co-expression is shown (yellow) in the cerebral cortex of control (arrowheads indicate non-neuronal apoptotic staining), hypoxic-ischaemic in the ipsilateral (arrows indicate neuronal apoptotic staining) and contralateral region (arrows indicate neuronal apoptotic staining), and intrauterine inflammation model 
(arrows and arrowheads indicate neuronal and non-neuronal apoptotic staining, repsectively). b, c Graphs (b) show the statistical significance in cleaved caspase-3 apoptosis and (c) show no statistical significance in neuronal cell counts co-expressing cleaved caspase-3. All values are mean with SEM. $(n=$ 3- 6 per group). ns, not significant; ** and $* \star \star$ denote significant $p<0.01$ and $p<0.001$, respectively using ANOVA with Dunnett's multiple comparisons test. Scale bar $=50 \mathrm{~mm}$. CTL, control; $\mathrm{HI}$, hypoxic-ischaemic; ipsi, ispsilateral side; contra, contralateral side; IUI, intrauterine inflammation.
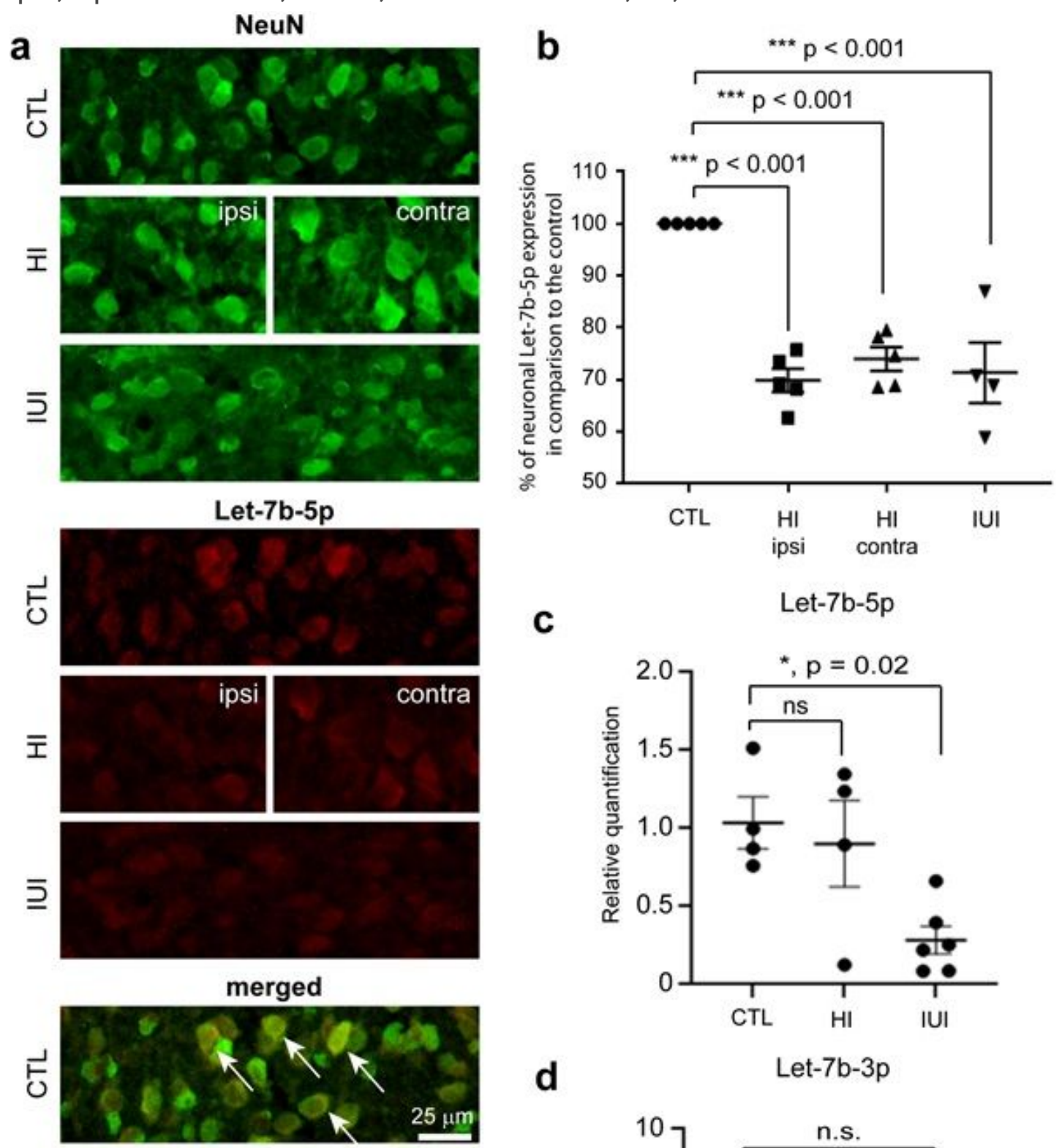

c

$$
\text { Let-7b-5p }
$$

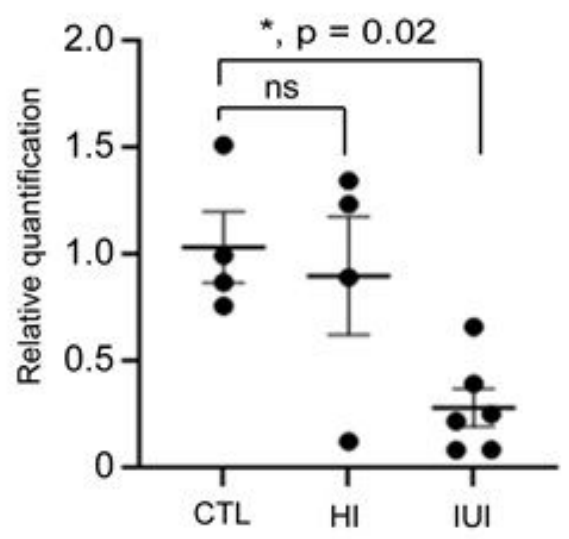

d

Let-7b-3p

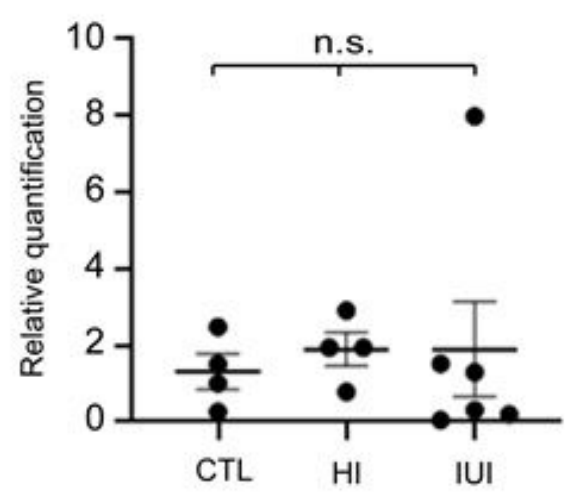

Figure 6 
Expression of let-7b-5p in the cerebral cortex in all animal models. a Images of NeuN positive neuronal cells (green) with let-7b-5p expression (red) and co-expression is shown (yellow) in the cerebral cortex of control (arrows indicate coexpression), hypoxic-ischaemic in the ipsilateral and contralateral region and intrauterine inflammation model. b Graph showing the statistical significance between the neonatal encephalopathy models vs control groups ( $n=4-6$ per group). $c-d$ Relative expression (2- $\Delta \Delta C t)$ of let-7b-5p (c) and let-7b-3p (d) in peripheral blood in the form of DBS from the rat animal models. All values are mean with SEM. ns, not significant, * and $* \star \star$ denote significant $p<0.05$ and $p<0.001$, respectively using ANOVA with Dunnett's multiple comparisons test. Scale bar $=25 \mathrm{~mm}$. CTL, control; HI, hypoxic-ischaemic; ipsi, ispsilateral side; contra, contralateral side; IUI, intrauterine inflammation. 


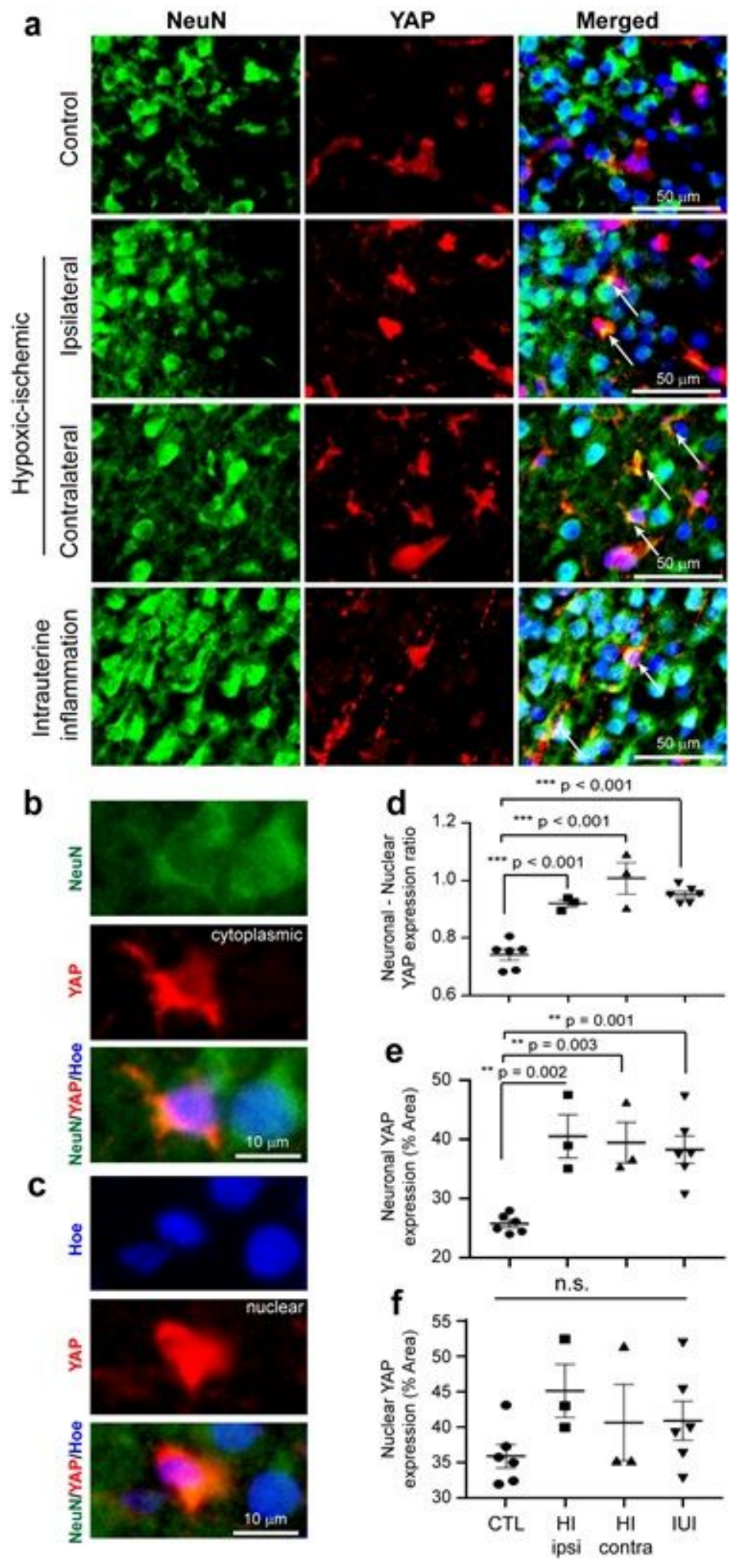

Figure 7

Neuronal expression of YAP in the cerebral cortex of the rat neonatal encephalopathy models. a Images of NeuN positive neuronal cells (green) with YAP (red) and co-expression is shown (yellow) in the cerebral cortex of control, hypoxic-ischaemic in the ipsilateral (arrows indicate YAP positive neurones) and contralateral region (arrows indicate YAP positive neurones), and intrauterine inflammation model (indicate YAP positive neurones). b Neuronal cytoplasmic YAP positive immunostaining is determined by 
strong co-expression of NeuN and YAP. c Nuclear YAP positive immunostaining is determined by strong co-expression of YAP with Hoechst staining in the nucleus. d-f Graphs showing significant increase in the neuronal/nuclear ratio (d), significant increase in neuronal (e), but no changes in nuclear (f) YAP

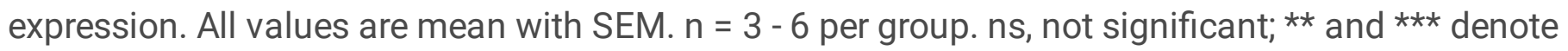
significant $p<0.01$ and $p<0.001$, respectively using ANOVA with Dunnett's multiple comparisons test. Scale bar $=50 \mathrm{~mm}$ for (a) and $10 \mathrm{~mm}$ for (b, c). CTL, control; HI, hypoxic-ischaemic; ipsi, ispsilateral side; contra, contralateral side; IUI, intrauterine inflammation.

\section{Supplementary Files}

This is a list of supplementary files associated with this preprint. Click to download.

- Supplementarydata.docx 